\title{
SPIN-LATTICE RELAXATION EFFECTS IN CRITICAL SOUND PROPAGATION
}

\author{
A. Pawlak \\ Institute of Physics, A. Mickiewicz University \\ Matejki 48/49, 60-769 Poznań, Poland
}

The nonasymptotic critical properties of sound propagation are studied in compressible Ising system above $T_{\mathrm{C}}$. In the present paper we analyse a model where in addition to the coupling to two order-parameter fluctuations the sound mode couples linearly to the fuctuations of spin-energy and lattice-energy densities. Both subsystems exchange energy with the rate determined by the bare spin-lattice relaxation time. The total energy may be conserved or not. The crossover between insulator-like behaviour $\sim t^{-2 \alpha}$ and metal-like behaviour $\sim t^{-(z \nu+\alpha)}$ in ultrasonic attenuation is investigated according to the value of ultrasonic frequency, the reduced temperature $t$, bare relaxation times and various coupling constants.

PACS numbers: 05.70.Jk, 62.65.+k

In typical ultrasonic experiments near the magnetic phase transition temperature we observe strong anomalies of sound attenuation in magnetic metals such as some rare-earth metals, whereas in magnetic insulators only very weak anomaly is observed [1, 2]. Many theories have been proposed to describe the strong anomaly in various substances [3-6]. They assume that the sound mode is coupled to two spin fluctuations above $T_{\mathrm{C}}$. On the other hand, the weak anomalies have been qualitatively explained by postulating the dominance of the linear coupling to the spin-energy density [7]. In our recent study we have investigated a model where both couplings were present [8]. In this paper we present more extensive discussion of the problem including also the lattice energy mode and transfer of energy between spin and lattice subsystems. We consider the acoustic phonon $Q$ coupled to the scalar spin $S$ and to the fluctuations of energy of spin and lattice subsystems. The IIamiltonian of the elastically isotropic system may be specified as

$$
\begin{aligned}
H= & \frac{1}{2} \int \mathrm{d}^{d} x\left[r S^{2}+(\nabla S)^{2}+u S^{4}+C_{12}\left(\sum_{\alpha} e_{\alpha \alpha}\right)^{2}+2 C_{44} \sum_{\alpha, \beta} e_{\alpha \beta}^{2}\right. \\
& \left.+g \sum_{\alpha} e_{\alpha \alpha} S^{2}+f e_{\mathrm{S}} S^{2}+w\left(e_{\mathrm{S}}+a e_{\mathrm{L}}\right) \sum_{\alpha} e_{\alpha \alpha}+\frac{e^{2} \mathrm{~S}}{C_{\mathrm{S}}}+\frac{e^{2} \mathrm{~J}}{C_{\mathrm{L}}}\right],
\end{aligned}
$$


where $e_{\alpha \beta}(x)$ denotes the strain tensor and $e_{\mathrm{S}}(x)$ and $e_{\mathrm{L}}(x)$ are the spin- and lattice-energy densities, respectively. The symbols $C_{\alpha \beta}$ stand for the bare elastic constants; $g, w, a w, f$ are the bare coupling constants, and $C_{\mathrm{S}}$ and $C_{\mathrm{L}}$ are the spin and lattice specific heats, respectively.

The dynamics of the system is described by the coupled Langevin equations

$$
\begin{aligned}
& \dot{S}=-\Gamma \frac{\delta H}{\delta S}+\xi, \\
& \ddot{Q}=-\frac{\delta H}{\delta Q}-\Gamma_{Q} \dot{Q}+\eta, \\
& \dot{e}_{\mathrm{S}}=-\left(\gamma_{\mathrm{S}}-\lambda_{\mathrm{S}} \nabla^{2}\right) \frac{\delta H}{\delta e_{\mathrm{S}}}+\gamma \frac{\delta H}{\delta e_{\mathrm{L}}}+\varphi, \\
& e_{\mathrm{L}}=-\left(\gamma_{\mathrm{L}}-\lambda_{\mathrm{S}} \nabla^{2}\right) \frac{\delta H}{\delta e_{\mathrm{L}}}+\gamma \frac{\delta H}{\delta e_{\mathrm{S}}}+\psi,
\end{aligned}
$$

where $\xi, \eta, \varphi$ and $\psi$ are Gaussian white noises with variances related to the bare damping terms $\Gamma, \Gamma_{Q},\left(\gamma_{\mathbf{S}}-\lambda_{\mathbf{S}} \nabla^{2}\right)$ and $\left(\gamma_{\mathrm{L}}-\lambda_{\mathbf{L}} \nabla^{2}\right)$ by the Einstein relations.

It is convenient to represent Eqs. (2)-(4) in functional form [9] with Lagrangian given by

$$
\begin{aligned}
& L\left(Q, \tilde{Q}, S, \tilde{S}_{,}, e_{\mathrm{S}}, \tilde{e}_{\mathrm{S}}, e_{\mathrm{L}}, \tilde{e}_{\mathrm{L}}\right)=\int_{\omega} \sum_{k}\left\{\Gamma \tilde{S}_{k, \omega} \tilde{S}_{-k,-\omega}+\Gamma_{\mathrm{Q}}(k) \tilde{Q}_{k, \omega} \tilde{Q}_{-k,-\omega}\right. \\
& +\Gamma_{\mathrm{S}}(k) \tilde{e}_{k, \omega} \mathrm{S}_{\tilde{e}_{k, \omega}} \mathrm{S}+\Gamma_{\mathrm{L}}(k) \tilde{e}_{k, \omega} \mathrm{L}_{\tilde{e}_{-k,-\omega}}{ }^{\mathrm{L}}-2 \gamma \tilde{e}_{k, \omega}^{\mathrm{S}} \tilde{e}_{-k,-\omega}^{\mathrm{L}} \\
& -\tilde{Q}_{k, \omega}\left[\left(-\omega^{2}+\mathrm{i} \omega\right) Q_{-k,-\omega}+\frac{\partial H}{\partial Q_{k, \omega}}\right]-\tilde{S}_{k, \omega}\left(\mathrm{i} \omega S_{-k,-\omega}+\Gamma \frac{\partial H}{\partial S_{k, \omega}}\right) \\
& -\tilde{e}_{k, \omega}^{\mathrm{S}}\left[\mathrm{i} \omega e_{-k,-\omega}^{\mathrm{S}}+\Gamma_{\mathrm{S}}(k) \frac{\partial H}{\partial e^{\mathrm{S}}{ }_{k, \omega}}-\gamma \frac{\partial H}{\partial e_{k, \omega}^{\mathrm{L}}}\right] \\
& \left.-\tilde{e}_{k, \omega}^{\mathrm{L}}\left[\mathrm{i} \omega e_{-k,-\omega}^{\mathrm{L}}+\Gamma_{\mathrm{L}}(k) \frac{\partial H}{\partial e^{\mathrm{L}} k, \omega}-\gamma \frac{\partial H}{\partial e_{k, \omega}^{\mathrm{S}}}\right]\right\}
\end{aligned}
$$

where $\Gamma_{i}(k)=\gamma_{i}+\lambda_{i} k^{2}$ for $i=\mathrm{S}, \mathrm{L}$ and $\tilde{S}, \tilde{Q}, \tilde{e_{S}}$ and $\tilde{e}_{\mathrm{L}}$ are auxiliary "response" fields. With the Lagrangian all correlation and response functions can be computed as path integrals weighted with density $\exp L$.

Next we apply series of Gaussian transformations

$$
e_{\mathrm{L}} \rightarrow e_{\mathrm{L}}+A e_{\mathrm{S}}+B \tilde{e}_{\mathrm{S}}, \quad \tilde{e}_{\mathrm{L}} \rightarrow \tilde{e}_{\mathrm{L}}+C \tilde{e}_{\mathrm{S}},
$$

which decouples the energy density modes

$$
\begin{aligned}
& Q \rightarrow Q+D S^{2}+E e_{\mathrm{S}}+F e_{\mathrm{L}}+G \widetilde{S^{2}}+H \tilde{e}_{\mathrm{S}}+J \tilde{e}_{\mathrm{L}}, \\
& \tilde{Q} \rightarrow \tilde{Q}+K \widetilde{S^{2}}+L \tilde{e}_{\mathrm{S}}+M \tilde{e}_{\mathrm{L}},
\end{aligned}
$$

decoupling the sound mode from the energy density modes, 


$$
\begin{aligned}
& e_{\mathrm{L}} \rightarrow e_{\mathrm{L}}+N S^{2}+P e_{\mathrm{S}}+R \widetilde{S^{2}}+T \tilde{e}_{\mathrm{S}} \\
& \tilde{e}_{\mathrm{L}} \rightarrow \tilde{e}_{\mathrm{L}}+U \widetilde{S^{2}}+V \tilde{e}_{\mathrm{S}}
\end{aligned}
$$

and

$$
\begin{aligned}
& e_{\mathrm{S}} \rightarrow e_{\mathrm{S}}+W S^{2}+X \widetilde{S^{2}}, \\
& \tilde{e}_{\mathrm{S}} \rightarrow \tilde{e}_{\mathrm{S}}+Y \widetilde{S^{2}}
\end{aligned}
$$

decoupling $e_{\mathrm{S}}$, e $e_{\mathrm{L}}$ and $S^{2}$, where $A, B, C \ldots$ are frequency and wave-vector dependent coefficients.

We then obtain expression for the acoustic self-energy

$$
\Sigma^{A C}=\frac{k^{2}\left[g^{2}(\hat{\omega})-\mathrm{i} \tilde{\omega} b(\hat{\omega}) g^{2}\right]\left\langle\Gamma \widetilde{S^{2}} S^{2}\right\rangle_{L^{e I t}}}{1-\mathrm{i} \tilde{\omega} b(\hat{\omega})\left(1+v\left\langle\Gamma \widetilde{S^{2}} S^{2}\right\rangle_{\mathrm{L}^{\mathrm{elI}}}\right)}
$$

where

$$
\begin{aligned}
& b(\hat{\omega})=\frac{1-\mathrm{i} \hat{\omega}}{m-\mathrm{i} \hat{\omega}}, \quad v=f^{2} C_{\mathrm{S}}, \quad m=1-\gamma^{2} / \Gamma_{\mathrm{S}} \Gamma_{\mathrm{L}} \\
& \tilde{\omega}=\omega C_{\mathrm{S}} / \Gamma_{\mathrm{S}}, \quad \hat{\omega}=\omega C_{\mathrm{L}} / \Gamma_{\mathrm{L}}
\end{aligned}
$$

and

$$
g(\hat{\omega})=g-w f C_{\mathrm{S}} \frac{m-\mathrm{i} \hat{\omega}\left(1-a \gamma / \Gamma_{\mathrm{S}}\right)}{m-\mathrm{i} \hat{\omega}}
$$

is a frequency-dependent effective spin-phonon coupling constant. The effective spin Lagrangian used for calculation of the expectation value in (11) contains strain- and energy density-mediated four-spin non-local interactions as a result of transformations (7)-(10). From the acoustic self-energy the sound attenuation coefficient can be easily found. Near the phase transition it can be written as

$$
\begin{aligned}
& \frac{\alpha(\omega, t)}{k^{2}} \propto\left\{\frac{1}{2 y \Gamma} \operatorname{Re}\left(g^{2}(\hat{\omega})\right) t^{-z \nu-\alpha} \operatorname{Im} \Phi(y)\right. \\
& \left.\quad+\frac{1}{2 y \Gamma} g^{2}|b(\hat{\omega})|^{2} \tilde{\omega}^{2} t^{-z \nu-\alpha} \operatorname{Im} \Phi(y)+\frac{C_{\mathrm{S}}}{\Gamma_{\mathrm{S}}} \operatorname{Re}\left[g^{2}(\hat{\omega}) \overline{b(\hat{\omega})}\right] t^{-2 \alpha}|\Phi(y)|^{2}\right\} \\
& \quad \times\left|1-\mathrm{i} \tilde{\omega} b(\hat{\omega}) t^{-\alpha} \Phi(y)\right|^{-2},
\end{aligned}
$$

where Re and Im denote real and imaginary part of a function, $t=\frac{T-T_{C}}{T_{C}}$ is the reduced temperature and $y=\frac{\omega}{2 \Gamma} t^{-z \nu}$ is the reduced frequency with $\alpha, \nu$ and $z$ being the critical exponents. The scaling function $\Phi(y)$ in the "weak-coupling" limit [5] can be written as

$$
\Phi(y)=\left[1+(y / 2)^{2}\right]^{-\alpha / 4 \nu}\left\{1+\frac{\mathrm{i} \alpha}{y \nu}\left[\mathrm{i}(1-\mathrm{i} y / 2) \arctan (y / 2)-\frac{1}{2} \ln \left(1+(y / 2)^{2}\right)\right]\right\} .
$$


Depending on the relative size of $t, \Gamma_{\mathrm{S}} / C_{\mathrm{S}}, \Gamma, \Gamma_{\mathrm{L}} / C_{\mathrm{L}}$ and $\omega$, Eq. (12) shows many different regimes. Asymptotically i.e. for $\omega, t \rightarrow 0$ strong singularity term always dominates $[3,10]$

$$
\alpha(\omega, l) \propto \omega^{2} t^{-(z \nu+\alpha)} \operatorname{Im} \Phi(y) / y .
$$

It is rather unexpected that a similar singularity dominates also for high frequencies, $\tilde{\omega} \gg 1$, where

$$
\alpha(\omega, t) \propto \omega^{2} t^{-(z \nu-\alpha)} \operatorname{Im} \Phi^{-1}(y) / y .
$$

This new behaviour can be obtained from the asymptotic behaviour by simple replacement $\alpha \rightarrow-\alpha$ and $\Phi \rightarrow \Phi^{-1}$. The weak singularity term,

$$
\alpha(\omega, t) \propto \omega^{2} t^{-2 \alpha}|\Phi(y)|^{2},
$$

can be dominant for $t>t_{\text {cross }}$ only if there exists a frequency window $\omega \ll$ $\Gamma_{\mathrm{S}} / C_{\mathrm{S}} \ll \Gamma$, with the crossover reduced temperature $t_{\mathrm{c}}$ ross $\propto\left(\frac{\alpha m \gamma_{\mathrm{S}}}{C_{\mathrm{S}} \Gamma}\right)^{1 /(z \nu-\alpha)}$. This regime is believed to take place in magnetic insulators $[1,2]$.

\section{References}

[1] K. Kawasaki, in: Phase Transilions and Critical Phenomena, Vol. 5a, Eds. C. Domb, M.S. Green, Academic Press, New York 1976, p. 165.

[2] B. Lüthi, in: Dynamical Properties of Solids, Vol. 3, Eds. G.K. Horton, A.A. Marudin, North-Holland, Amsterdam 1980, p. 245.

[3] K.K. Murata, Phys. Rev. B 13, 4015 (1976).

[4] J.O. Fossum, J. Phys. C, Solid State Phys. 18, 5531 (1985).

[5] A. Pawlak, J. Phys., Condens. Matter 1, 7989 (1989).

[6] A. Pawlak, Phys. Rev. B 44, 5296 (1991).

[7] K. Kawasaki, Plyys. Lett. A 29, 406 (1968).

[8] A. Pawlak, Acta Phys. Pol. A 85, 385 (1994).

[9] H.K. Janssen, Z. Phys. B 23, 377 (1976).

[10] II. Iro, F. Schwabl, Solid State Commun. 46, 205 (1983). 